\title{
EINSTEIN DOUBLY WARPED PRODUCT MANIFOLDS WITH SEMI-SYMMETRIC METRIC CONNECTION
}

\author{
By Punam Gupta and Abdoul Salam Diallo
}

\begin{abstract}
In this paper, we study the doubly warped product manifolds with semi-symmetric metric connection. We derive the curvature formulas for doubly warped product manifold with semi-symmetric metric connection in terms of curvatures of components of doubly warped product manifolds. We also prove the necessary and sufficient condition for a doubly warped product manifold to be a warped product manifold. We obtain some results for an Einstein doubly warped product manifold and Einstein-like doubly warped product manifold of class $\mathcal{A}$ with respect to a semi-symmetric metric connection.
\end{abstract}

1. Introduction. In 1969, Bishop and O'Neill [4] introduced singly warped products or warped products. They used this concept to construct Riemannian manifolds with negative sectional curvature. The warped product $B \times{ }_{h} F$ of two Riemannian manifolds $\left(B, g_{B}\right)$ and $\left(F, g_{F}\right)$ with a smooth function $h: B \rightarrow(0, \infty)$ is a product manifold of form $B \times F$ with the metric tensor $g=g_{B} \oplus h^{2} g_{F}$. Here, $\left(B, g_{B}\right)$ is called the base manifold, $\left(F, g_{F}\right)$ is called the fiber manifold and $h$ is called the warping function. In 1983, O'Neill [16] discussed warped products and derived curvature formulas of warped products in terms of curvatures of components of warped products.

In general, doubly warped products are generalization of singly warped products. The doubly warped product $B_{f} \times{ }_{h} F$ of two Riemannian manifolds $\left(B, g_{B}\right)$ and $\left(F, g_{F}\right)$ with smooth functions, which are known as warping functions, $h: B \rightarrow(0, \infty), f: F \rightarrow(0, \infty)$ is a product manifold of form $B \times F$ with the metric tensor $g=f^{2} g_{B} \oplus h^{2} g_{F}$. Doubly warped products are

2010 Mathematics Subject Classification. 53C05, 53C25, 53C50.

Key words and phrases. Doubly warped products, semi-symmetric metric connection, Levi-Civita connection, Einstein manifolds, Einstein-like manifold of class $\mathcal{A}$. 
studied by many authors: Allison [2] studied pseudoconvexity of Lorentzian doubly warped products; Gebarowski considered conformal properties of doubly warped products in $([\mathbf{8}],[\mathbf{9}])$. Geodesic completeness of Riemannian and Lorentzian doubly warped products have been studied by Unal [25]. In 2003, Ramos et al. [19] gave an invariant characterization of doubly warped spacetimes in terms of Newman-Penrose formalism, and proposed a classification scheme. Recently, the first author [10] studied the compact Einstein doubly warped product manifold and established some interesting results.

In 1924, Friedmann and Schouten [7] introduced the idea of a semi-symmetric linear connection on a Riemannian manifold. Later, in 1932, Hayden [12] introduced the concept of semi-symmetric metric connection. A metric connection is a Levi-Civita connection when its torsion is zero and it becomes the Hayden connection [12] when it has a non-zero torsion. Thus, metric connections include both Levi-Civita connections and Hayden connections. In 1970, Yano [26] considered the semi-symmetric metric connection and studied some of its properties.

In 2001, Sular and Özgur [21] studied the warped product with semisymmetric metric connection and established some results. Motivated by these results, we study doubly warped product manifolds with a semi-symmetric metric connection and derive the curvature formulas for doubly warped product manifolds with semi-symmetric metric connection in terms of curvatures of components of doubly warped product manifolds. We also obtain results for Einstein doubly warped product manifold and Einstein-like doubly warped product manifold of class $\mathcal{A}$ with respect to a semi-symmetric metric connection.

2. Preliminaries. Let $M$ be an $n$-dimensional Riemannian manifold with Riemannian metric $g$. A linear connection $\tilde{\nabla}$ on a Riemannian manifold $M$ is called a semi-symmetric connection if the torsion tensor $\tilde{T}$ of the connection $\tilde{\nabla}$ given by

$$
\tilde{T}(X, Y)=\tilde{\nabla}_{X} Y-\tilde{\nabla}_{Y} X-[X, Y]
$$

satisfies

$$
\tilde{T}(X, Y)=\pi(Y) X-\pi(X) Y,
$$

where $\pi$ is a 1-form associated with the vector field $P$ on $M$ defined by

$$
\pi(X)=g(X, P) .
$$

A semi-symmetric connection $\tilde{\nabla}$ is called a semi-symmetric metric connection if $\tilde{\nabla} g=0$. Let $\nabla$ be the Levi-Civita connection of a Riemannian manifold, then the unique semi-symmetric metric connection $\tilde{\nabla}$ given by Yano $[\mathbf{2 6}]$ is

$$
\tilde{\nabla}_{X} Y=\nabla_{X} Y+\pi(Y) X-g(X, Y) P .
$$


A relation between the curvature tensors $R$ and $\tilde{R}$ of the Levi-Civita connection $\nabla$ and the semi-symmetric connection $\tilde{\nabla}$ is given by

$$
\begin{aligned}
\tilde{R}(X, Y) Z= & R(X, Y) Z+g\left(Z, \nabla_{X} P\right) Y-g\left(Z, \nabla_{Y} P\right) X \\
& +g(X, Z) \nabla_{Y} P-g(Y, Z) \nabla_{X} P \\
& +\pi(P)(g(X, Z) Y-g(Y, Z) X) \\
& +(g(Y, Z) \pi(X)-g(X, Z) \pi(Y)) P \\
& +\pi(Z)(\pi(Y) X-\pi(X) Y), \quad X, Y, Z \in \mathfrak{X}(M),
\end{aligned}
$$

where $\mathfrak{X}(M)$ is the set of smooth vector fields on $M[26]$. Yano proved that a Riemannian manifold is conformally flat if and only if it admits a semisymmetric metric connection whose curvature tensor vanishes identically. This result was generalized onto vanishing Ricci tensor of the semi-symmetric metric connection by T. Imai (see [13], [14]). For a general survey of various kinds of connections (see Tripathi $[\mathbf{2 4}]$ ).

3. Doubly Warped Product Manifolds. Doubly warped product manifolds were introduced as a generalization of warped product manifolds. Let $\left(B, g_{B}\right)$ and $\left(F, g_{F}\right)$ be two Riemannian manifolds with real dimension $n_{1}$ and $n_{2}$, respectively. Let $h: B \rightarrow \mathbb{R}^{+}$and $f: F \rightarrow \mathbb{R}^{+}$be two smooth functions. Consider the product manifold $B \times F$ with its projections $\rho: B \times F \rightarrow B$ and $\sigma: B \times F \rightarrow F$. The doubly warped product $B_{f} \times{ }_{h} F$ is the product manifold $B \times F$ furnished with the metric tensor

$$
g=(f \circ \sigma)^{2} \rho^{*}\left(g_{B}\right)+(h \circ \rho)^{2} \sigma^{*}\left(g_{F}\right),
$$

where $^{*}$ denotes pullback. If $X$ is tangent to $B \times F$ at $(p, q)$, then

$$
g(X, X)=f^{2}(q) g_{B}(d \rho(X), d \rho(X))+h^{2}(p) g_{F}(d \sigma(X), d \sigma(X)) .
$$

Thus, we have

$$
g=f^{2} g_{B}+h^{2} g_{F} .
$$

The functions $h$ and $f$ are called the warping functions of the doubly warped product. The manifold $B$ is called the base of $(M, g)$ and the manifold $F$ is called the fibre of $(M, g)$. If the warping function $f$ or $h$ is constant, then the doubly warped product $B_{f} \times{ }_{h} F$ reduces to a warped product $B \times{ }_{h} \tilde{F}$ (or $\tilde{B}_{f} \times F$ ), where the fibre $\tilde{F}$ is just $F$ with metric $\tilde{g}_{F}$ given by $\frac{1}{h^{2}} g_{F}$ (or where the base $\tilde{B}$ is just $B$ with metric $\tilde{g}_{B}$ given by $\left.\frac{1}{f^{2}} g_{B}\right)$. If the warping function $f$ or $h$ is equal to 1 , then the doubly warped product $B_{f} \times{ }_{h} F$ reduces to a warped product $B \times{ }_{h} F\left(\right.$ or $\left.B_{f} \times F\right)$. If both $f$ and $h$ are constant, then it is simply a product manifold $\tilde{B} \times \tilde{F}$, where the fibre $\tilde{F}$ is just $F$ with metric $\tilde{g}_{F}$ given by $\frac{1}{h^{2}} g_{F}$ and the base $\tilde{B}$ is just $B$ with metric $\tilde{g}_{B}$ given by $\frac{1}{f^{2}} g_{B}$. If both $f$ and $h$ are equal to 1 , then it is simply a product manifold $B \times F$. 
The sets of all smooth and positive valued functions $h: B \rightarrow \mathbb{R}^{+}$and $f: F \rightarrow \mathbb{R}^{+}$are denoted by $\mathcal{F}(B)=C^{\infty}(B)$ and $\mathcal{F}(F)=C^{\infty}(F)$, respectively. The lifts of $h$ and $f$ to $M$ are defined by $\tilde{h}=h \circ \rho \in \mathcal{F}(M)$ and $\tilde{f}=f \circ \sigma \in$ $\mathcal{F}(M)$, respectively. If $X_{p} \in T_{p}(B)$ and $q \in F$, then the lift $\tilde{X}_{(p, q)}$ of $X_{p}$ to $M$ is the unique tangent vector in $T_{(p, q)}(B \times\{q\})$ such that $d \rho_{(p, q)}\left(\tilde{X}_{(p, q)}\right)=X_{p}$ and $d \sigma_{(p, q)}\left(\tilde{X}_{(p, q)}\right)=0$. The set of all such horizontal tangent vector lifts will be denoted by $L_{(p, q)}(B)$. Similarly, we can define the set of all vertical tangent vector lifts $L_{(p, q)}(F)$.

Let $X \in \mathfrak{X}(B)$, where $\mathfrak{X}(B)$ is the set of smooth vector fields on $B$. The lift $\tilde{X}$ of $X$ to $M$ is the unique element of $\mathfrak{X}(M)$ whose value, at each $(p, q)$, is the lift of $X_{p}$ to $(p, q)$. The set of such lifts will be denoted by $\mathcal{L}(B)$. In a similar manner, we can define $\mathcal{L}(F)$. Throughout the paper, we assume that $X, Y, Z \in \mathcal{L}(B)$ and $U, V, W \in \mathcal{L}(F)$. The connections $\nabla,{ }^{B} \nabla$ and ${ }^{F} \nabla$ are Levi-Civita connections on $M, B$ and $F$, respectively. We will denote by $R,{ }^{B} R$ and ${ }^{F} R$ the Riemann curvatures on $M, B$ and $F$, respectively; $S,{ }^{B} S$ and ${ }^{F} S$ the Ricci tensors for the connections $\nabla,{ }^{B} \nabla$ and ${ }^{F} \nabla$, respectively; $r,{ }^{B} r$ and ${ }^{F} r$ are the scalar curvatures for the connections $\nabla,{ }^{B} \nabla$ and ${ }^{F} \nabla$, respectively.

We need the following lemmas for later use. For more details see $[\mathbf{1}, \mathbf{1 1}$, $18,25]$.

Lemma 3.1. Let $M=B_{f} \times{ }_{h} F$ be a doubly warped product manifold. If $X, Y \in \mathfrak{X}(B)$ and $V, W \in \mathfrak{X}(F)$, then

$$
\begin{gathered}
\tan \nabla_{X} Y=-\frac{\operatorname{grad} f}{f} g(X, Y), \\
\text { nor } \nabla_{X} Y \text { is the lift of } \nabla_{X} Y \text { on } B, \\
\nabla_{X} V=\nabla_{V} X=\frac{X h}{h} V+\frac{V f}{f} X, \\
\operatorname{nor} \nabla_{V} W=-\frac{\operatorname{grad} h}{h} g(V, W), \\
\tan \nabla_{V} W \text { is the lift of } \nabla_{V} W \text { on } F,
\end{gathered}
$$

where tan and nor stand for tangent part to $F$ and normal part to $B$, respectively.

Lemma 3.2. Let $M=B_{f} \times{ }_{h} F$ be a doubly warped product manifold. If $X, Y, Z \in \mathfrak{X}(B)$ and $U, V, W \in \mathfrak{X}(F)$, then

$$
R(X, Y) Z={ }^{B} R(X, Y) Z+\frac{\|\operatorname{grad} f\|^{2}}{f^{2}}(g(X, Z) Y-g(Y, Z) X),
$$




$$
\begin{aligned}
R(X, V) Y & =\frac{H_{B}^{h}(X, Y)}{h} V+\frac{1}{f} g(X, Y)^{F} \nabla_{V}(\operatorname{grad} f), \\
R(X, Y) V & =\frac{V f}{f}\left(\frac{Y h}{h} X-\frac{X h}{h} Y\right), \\
R(X, V) W & =-\frac{H_{F}^{f}(V, W)}{f} X-\frac{1}{h} g(V, W)^{B} \nabla_{X}(\operatorname{grad} h), \\
R(V, W) X & =\frac{X h}{h}\left(\frac{W f}{f} V-\frac{V f}{f} W\right), \\
R(V, W) U & ={ }^{F} R(V, W) U+\frac{\|\operatorname{grad} h\|^{2}}{h^{2}}(g(V, U) W-g(W, U) V),
\end{aligned}
$$

where $H_{B}^{h}$ and $H_{F}^{f}$ are the Hessian of $h$ and $f$, respectively.

Lemma 3.3. Let $M=B_{f} \times{ }_{h} F$ be a doubly warped product manifold. If $X, Y \in \mathfrak{X}(B)$ and $V, W \in \mathfrak{X}(F)$, then

$$
\begin{aligned}
S(X, Y)= & { }^{B} S(X, Y)-\frac{n_{2}}{h} H_{B}^{h}(X, Y) \\
& -g(X, Y)\left(\left(n_{1}-1\right) \frac{\|\operatorname{grad} f\|^{2}}{f^{2}}+\frac{\Delta_{F} f}{f}\right), \\
S(X, V)= & \frac{(n-2)(X h)(V f)}{h f}, \\
S(V, W)= & { }^{F} S(V, W)-\frac{n_{1}}{f} H_{F}^{f}(V, W) \\
& -g(V, W)\left(\left(n_{2}-1\right) \frac{\|\operatorname{grad} h\|^{2}}{h^{2}}+\frac{\Delta_{B} h}{h}\right),
\end{aligned}
$$

where $\Delta_{F} f$ and $\Delta_{B}$ h are the Laplacian of $f$ on $F$ and $h$ on $B$, respectively.

Lemma 3.4. Let $M=B_{f} \times{ }_{h} F$ be a doubly warped product manifold. Then

$$
\begin{aligned}
r= & \frac{1}{f^{2}} B_{r}+\frac{1}{h^{2}} F_{r}-2 n_{1} \frac{\Delta_{F} f}{f}-2 n_{2} \frac{\Delta_{B} h}{h} \\
& -n_{1}\left(n_{1}-1\right) \frac{\|\operatorname{grad} f\|^{2}}{f^{2}}-n_{2}\left(n_{2}-1\right) \frac{\|\operatorname{grad} h\|^{2}}{h^{2}} .
\end{aligned}
$$

4. Doubly Warped Product Manifolds Endowed with the SemiSymmetric Metric Connection. In this section, we consider doubly warped product manifolds with respect to the semi-symmetric metric connection and find new expressions concerning curvature tensor, Ricci tensor and the scalar curvature admitting the semi-symmetric metric connection where the associated vector field $P \in \mathfrak{X}(B)$ or $P \in \mathfrak{X}(F)$. 
The following results hold true.

Proposition 4.1. Let $M=B_{f} \times{ }_{h} F$ be a doubly warped product manifold and let $\tilde{\nabla},{ }^{B} \tilde{\nabla}$ and ${ }^{F} \tilde{\nabla}$ be the semi-symmetric metric connections on $M, B$ and $F$, respectively. If $X, Y \in \mathfrak{X}(B), V, W \in \mathfrak{X}(F)$ and $P \in \mathfrak{X}(B)$, then

$$
\begin{gathered}
\tan \tilde{\nabla}_{X} Y=-\frac{1}{f} g(X, Y) \operatorname{grad} f, \\
\operatorname{nor} \tilde{\nabla}_{X} Y \text { is the lift of }{ }^{B} \tilde{\nabla}_{X} Y, \\
\tilde{\nabla}_{X} V=\frac{X h}{h} V+\frac{V f}{f} X, \\
\tilde{\nabla}_{V} X=\frac{X h}{h} V+\frac{V f}{f} X+\pi(X) V, \\
\operatorname{nor} \tilde{\nabla}_{V} W=-\left(\frac{\operatorname{grad} h}{h}+P\right) g(V, W), \\
\tan \tilde{\nabla}_{V} W \text { is the lift of } F \tilde{\nabla}_{V} W \text { on } F,
\end{gathered}
$$

where tan and nor stand for tangent part to $F$ and normal part to $B$, respectively.

Proof. Considering $P \in \mathfrak{X}(B)$ and using (2.4), first equation of Lemma 3.1, we get (4.1). Simailarly, we can easily find the others statements by using (2.4) and Lemma 3.1.

Proposition 4.2. Let $M=B_{f} \times{ }_{h} F$ be a doubly warped product manifold and let $\tilde{\nabla},{ }^{B} \tilde{\nabla}$ and ${ }^{F} \tilde{\nabla}$ be the semi-symmetric metric connections on $M, B$ and $F$, respectively. If $X, Y \in \mathfrak{X}(B), V, W \in \mathfrak{X}(F)$ and $P \in \mathfrak{X}(F)$, then

$$
\begin{gathered}
\tan \tilde{\nabla}_{X} Y=-\left(\frac{\operatorname{grad} f}{f}+P\right) g(X, Y), \\
\text { nor } \tilde{\nabla}_{X} Y \text { is the lift of }{ }^{B} \tilde{\nabla}_{X} Y, \\
\tilde{\nabla}_{X} V=\frac{X h}{h} V+\frac{V f}{f} X+\pi(V) X, \\
\tilde{\nabla}_{V} X=\frac{X h}{h} V+\frac{V f}{f} X, \\
\operatorname{nor} \tilde{\nabla}_{V} W=-\frac{\operatorname{grad} h}{h} g(V, W), \\
\tan \tilde{\nabla}_{V} W \text { is the lift of } F \tilde{\nabla}_{V} W \text { on } F .
\end{gathered}
$$

Proof. Considering $P \in \mathfrak{X}(F)$ and using (2.4), first equation of Lemma 3.1 , we get (4.7). Simailarly, we can easily find the others results by using (2.4) and Lemma 3.1 . 
Proposition 4.3. Let $M=B_{f} \times{ }_{h} F$ be a doubly warped product manifold and let $\tilde{R},{ }^{B} \tilde{R}$ and ${ }^{F} \tilde{R}$ be the Riemannian curvature tensors with respect to the semi-symmetric metric connections $\tilde{\nabla},{ }^{B} \tilde{\nabla}$ and ${ }^{F} \tilde{\nabla}$, respectively. If $X, Y, Z \in$ $\mathfrak{X}(B), U, V, W \in \mathfrak{X}(F)$ and $P \in \mathfrak{X}(B)$, then

$$
\begin{aligned}
& \tilde{R}(X, Y) Z={ }^{B} \tilde{R}(X, Y) Z+\frac{\|\operatorname{grad} f\|^{2}}{f^{2}}(g(X, Z) Y-g(Y, Z) X) \\
& +(g(Y, Z) \pi(X)-g(X, Z) \pi(Y)) \frac{\operatorname{grad} f}{f}, \\
& \tilde{R}(V, X) Y=\left(\frac{H_{B}^{h}(X, Y)}{h}+\pi(X) \pi(Y)-g\left(Y{ }^{B} \nabla_{X} P\right)\right) V+\frac{V f}{f} \pi(Y) X \\
& -\left(\frac{V f}{f} P+\frac{P h}{h} V+\pi(P) V-\frac{1}{f}^{F} \nabla_{V} \operatorname{grad} f\right) g(X, Y), \\
& \tilde{R}(X, Y) V=\left(\frac{(V f)(Y h)}{h f}+\frac{(V f)}{f} \pi(Y)\right) X \\
& -\left(\frac{(V f)(X h)}{h f}+\frac{(V f)}{f} \pi(X)\right) Y, \\
& \tilde{R}(V, W) X=\left(\frac{(W f)(X h)}{h f}-\frac{(W f)}{f} \pi(X)\right) V \\
& -\left(\frac{(V f)(X h)}{h f}-\frac{(V f)}{f} \pi(X)\right) W, \\
& \tilde{R}(X, V) W=-\frac{H_{F}^{f}(V, W)}{f} X-\frac{W f}{f} \pi(X) V \\
& -g(V, W)\left(\frac{{ }^{B} \nabla_{X} \operatorname{grad} h}{h}+\frac{P h}{h} X+{ }^{B} \nabla_{X} P\right. \\
& \left.+\pi(P) X-\pi(X) P-\frac{\operatorname{grad} f}{f} \pi(X)\right), \\
& \tilde{R}(U, V) W={ }^{F} R(U, V) W-\frac{U f}{f} g(V, W) P+\frac{V f}{f} g(U, W) P \\
& -\left(\frac{\|\operatorname{grad} h\|^{2}}{h^{2}}+\frac{2 P h}{h}+\pi(P)\right)(g(V, W) U-g(U, W) V) .
\end{aligned}
$$

Proof. Assume that $M=B_{f} \times{ }_{h} F$ is a doubly warped product and $R$ and $\tilde{R}$ denote the curvature tensors with respect to the Levi-Civita connection and the semi-symmetric metric connection, respectively. 
In view of the (2.5), Lemma 3.1 and Lemma 3.2, we can write

$$
\begin{aligned}
\tilde{R}(X, Y) Z= & { }^{B} R(X, Y) Z+\frac{\|\operatorname{grad} f\|^{2}}{f^{2}}(g(X, Z) Y-g(Y, Z) X) \\
& +g\left(Z,{ }^{B} \nabla_{X} P\right) Y-g\left(Z,{ }^{B} \nabla_{Y} P\right) X \\
& +g(X, Z)\left({ }^{B} \nabla_{Y} P+\pi(P) Y-\pi(Y) P\right) \\
& -g(Y, Z)\left({ }^{B} \nabla_{X} P+\pi(P) X-\pi(X) P\right) \\
& +(g(Y, Z) \pi(X)-g(X, Z) \pi(Y)) \frac{\operatorname{grad} f}{f} \\
& +\pi(Z)(\pi(Y) X-\pi(X) Y) .
\end{aligned}
$$

By using equation (2.5), we can write

$$
\begin{aligned}
\tilde{R}(V, X) Y= & R(V, X) Y+g\left(Y, \nabla_{V} P\right) X-g\left(Y, \nabla_{X} P\right) V \\
& -g(X, Y)\left[\nabla_{V} P+\pi(P) V-\pi(V) P\right] \\
& +\pi(Y)[\pi(X) V-\pi(V) X] .
\end{aligned}
$$

Since $P \in \mathfrak{X}(B)$ and by making use of Lemma 3.1 and Lemma 3.2, we get

$$
\begin{aligned}
\tilde{R}(V, X) Y= & \left(\frac{H_{B}^{h}(X, Y)}{h}+\pi(X) \pi(Y)-g\left(Y{ }^{B} \nabla_{X} P\right)\right) V+\frac{V f}{f} \pi(Y) X \\
& -\left(\frac{V f}{f} P+\frac{P h}{h} V+\pi(P) V-\frac{1}{f} F^{F} \nabla_{V} \operatorname{grad} f\right) g(X, Y) .
\end{aligned}
$$

Replacing $Z$ with $V$ in equation (2.5), we get

$$
\tilde{R}(X, Y) V=R(X, Y) V+g\left(V, \nabla_{X} P\right) Y-g\left(V, \nabla_{Y} P\right) X .
$$

Using Lemma 3.1 and Lemma 3.2, we get

$$
\begin{aligned}
\tilde{R}(X, Y) V= & \left(\frac{(V f)(Y h)}{h f}+\frac{(V f)}{f} \pi(Y)\right) X \\
& -\left(\frac{(V f)(X h)}{h f}+\frac{(V f)}{f} \pi(X)\right) Y .
\end{aligned}
$$

By making use of (2.5) and Lemma 3.1 Lemma 3.2, we get

$$
\begin{aligned}
\tilde{R}(V, W) X= & \left(\frac{(W f)(X h)}{h f}-\frac{(W f)}{f} \pi(X)\right) V \\
& -\left(\frac{(V f)(X h)}{h f}-\frac{(V f)}{f} \pi(X)\right) W .
\end{aligned}
$$


From the equation (2.5), we find

$$
\begin{aligned}
\tilde{R}(X, V) W= & R(X, V) W+g\left(W, \nabla_{X} P\right) V-g\left(W, \nabla_{V} P\right) X \\
& -g(V, W)\left(\nabla_{X} P+\pi(P) X-\pi(X) P\right) .
\end{aligned}
$$

Using Lemma 3.1 and Lemma 3.2 in (4.16), we have

$$
\begin{aligned}
\tilde{R}(X, V) W= & -\frac{H_{F}^{f}(V, W)}{f} X-\frac{W f}{f} \pi(X) V \\
& -g(V, W)\left(\frac{{ }^{B} \nabla_{X} \operatorname{grad} h}{h}+\frac{P h}{h} X+{ }^{B} \nabla_{X} P\right. \\
& \left.+\pi(P) X-\pi(X) P-\frac{\operatorname{grad} f}{f} \pi(X)\right) .
\end{aligned}
$$

In view of equation (2.5), we have

$$
\begin{aligned}
\tilde{R}(U, V) W= & R(U, V) W+g\left(W, \nabla_{U} P\right) V-g\left(W, \nabla_{V} P\right) U \\
& +g(U, W) \nabla_{V} P-g(V, W) \nabla_{U} P \\
& +\pi(P)[g(U, W) V-g(V, W) U] .
\end{aligned}
$$

By making use of Lemma 3.1 and Lemma 3.2 in (4.17), we obtain

$$
\begin{aligned}
\tilde{R}(U, V) W= & { }^{F} R(U, V) W-\frac{U f}{f} g(V, W) P+\frac{V f}{f} g(U, W) P \\
& -\left(\frac{\|\operatorname{grad} h\|^{2}}{h^{2}}+\frac{2 P h}{h}+\pi(P)\right)(g(V, W) U-g(U, W) V) .
\end{aligned}
$$

Hence, the proof is completed.

Proposition 4.4. Let $M=B_{f} \times{ }_{h} F$ be a doubly warped product manifold and let $\tilde{R},{ }^{B} \tilde{R}$ and ${ }^{F} \tilde{R}$ be the Riemannian curvature tensors with respect to the semi-symmetric metric connections $\tilde{\nabla},{ }^{B} \tilde{\nabla}$ and ${ }^{F} \tilde{\nabla}$, respectively. If $X, Y, Z \in$ $\mathfrak{X}(B), U, V, W \in \mathfrak{X}(F)$ and $P \in \mathfrak{X}(F)$, then

$$
\begin{aligned}
\tilde{R}(X, Y) Z= & { }^{B} R(X, Y) Z+\left(g(X, Z) \frac{Y h}{h}-g(Y, Z) \frac{X h}{h}\right)\left(P-\frac{\operatorname{grad} f}{f}\right) \\
& +\left(\frac{\|\operatorname{grad} f\|^{2}}{f^{2}}+\frac{2 P f}{f}+\pi(P)\right)(g(X, Z) Y-g(Y, Z) X), \\
\tilde{R}(V, X) Y= & -\left(\frac{H_{B}^{h}(X, Y)}{h}+\frac{P f}{f} g(X, Y)+\pi(P) g(X, Y)\right) V-\frac{(Y h)}{h} \pi(V) X \\
& +g(X, Y)\left(\frac{1}{h} \pi(V) \operatorname{grad} h+\pi(V) P-{ }^{F} \nabla_{V} P-\frac{1}{f}{ }^{F} \nabla_{V} \operatorname{grad} f\right),
\end{aligned}
$$




$$
\begin{aligned}
\tilde{R}(X, Y) V= & \left(\frac{(V f)(Y h)}{h f}-\frac{(Y h)}{h} \pi(V)\right) X-\left(\frac{(V f)(X h)}{h f}-\frac{(X h)}{h} \pi(V)\right) Y, \\
\tilde{R}(V, W) X= & \left(\frac{(W f)(X h)}{h f}+\frac{(X h)}{h} \pi(W)\right) V-\left(\frac{(V f)(X h)}{h f}+\frac{(X h)}{h} \pi(V)\right) W, \\
\tilde{R}(X, V) W= & -g(V, W)\left(\frac{1}{h}^{B} \nabla_{X} \operatorname{grad} h+\frac{P f}{f} X+\pi(P) X+\frac{(X h)}{h} P\right) \\
& -\left(\frac{H_{F}^{f}(V, W)}{f}-\pi(V) \pi(W)+g\left(W,^{F} \nabla_{V} P\right)\right) X+\frac{(X h)}{h} \pi(W) V,
\end{aligned}
$$

$$
\begin{aligned}
\tilde{R}(U, V) W= & { }^{F} \tilde{R}(U, V) W-\frac{\|\operatorname{grad} h\|^{2}}{h^{2}}(g(V, W) U-g(U, W) V) \\
& +(\pi(U) g(V, W)-g(U, W) \pi(V)) \frac{\operatorname{grad} h}{h}
\end{aligned}
$$

Proof. Assume that the associated vector field $P$ is in $\mathfrak{X}(F)$. Then the equation (2.5) can be written as

$$
\begin{aligned}
\tilde{R}(X, Y) Z= & R(X, Y) Z+g\left(Z, \nabla_{X} P\right) Y-g\left(Z, \nabla_{Y} P\right) X \\
& +g(X, Z) \nabla_{Y} P-g(Y, Z) \nabla_{X} P \\
& +\pi(P)(g(X, Z) Y-g(Y, Z) X) .
\end{aligned}
$$

By the use of Lemma 3.1 and Lemma 3.2, the above equation gives us

$$
\begin{aligned}
\tilde{R}(X, Y) Z= & { }^{B} R(X, Y) Z \\
& +\left(\frac{\|\operatorname{grad} f\|^{2}}{f^{2}}+\frac{2 P f}{f}+\pi(P)\right)(g(X, Z) Y-g(Y, Z) X) \\
& +(g(Y, Z)(X h)-g(X, Z)(Y h))\left(\frac{\operatorname{grad} f}{h f}-\frac{P}{h}\right) .
\end{aligned}
$$

By (4.13), Lemma 3.1 and Lemma 3.2, we obtain

$$
\begin{aligned}
\tilde{R}(V, X) Y= & -\left(\frac{H_{B}^{h}(X, Y)}{h}+\frac{P f}{f} g(X, Y)+\pi(P) g(X, Y)\right) V-\frac{(Y h)}{h} \pi(V) X \\
& +g(X, Y)\left(\frac{1}{h} \pi(V) \operatorname{grad} h+\pi(V) P-{ }^{F} \nabla_{V} P-\frac{1}{f}{ }^{F} \nabla_{V} \operatorname{grad} f\right),
\end{aligned}
$$

Replacing $Z$ with $V$ in equation (2.5), we get

$$
\tilde{R}(X, Y) V=R(X, Y) V+g\left(V, \nabla_{X} P\right) Y-g\left(V, \nabla_{Y} P\right) X .
$$


Using Lemma 3.1 and Lemma 3.2, we obtain

$$
\begin{aligned}
\tilde{R}(X, Y) V= & \left(\frac{(V f)(Y h)}{h f}-\frac{Y h}{h} \pi(V)\right) X \\
& -\left(\frac{(V f)(X h)}{h f}-\frac{X h}{h} \pi(V)\right) Y .
\end{aligned}
$$

From equation (2.5), we get

$$
\tilde{R}(V, W) X=R(V, W) X+g\left(X, \nabla_{V} P\right) W-g\left(X, \nabla_{W} P\right) V .
$$

By Lemma 3.1 and Lemma 3.2, we get

$$
\begin{aligned}
\tilde{R}(V, W) X= & \left(\frac{(W f)(X h)}{h f}+\frac{(X h)}{h} \pi(W)\right) V \\
& -\left(\frac{(V f)(X h)}{h f}-\frac{(X h)}{h} \pi(V)\right) W .
\end{aligned}
$$

From equation (2.5), we get

$$
\begin{aligned}
\tilde{R}(X, V) W= & R(X, V) W+g\left(W, \nabla_{X} P\right) V-g\left(W, \nabla_{V} P\right) X \\
& -g(V, W) \nabla_{X} P-\pi(P) g(V, W) X+\pi(V) \pi(W) X .
\end{aligned}
$$

Using Lemma 3.1 and Lemma 3.2, we get

$$
\begin{aligned}
\tilde{R}(X, V) W= & -g(V, W)\left(\frac{1}{h}^{B} \nabla_{X} \operatorname{grad} h+\frac{P f}{f} X+\pi(P) X+\frac{(X h)}{h} P\right) \\
& -\left(\frac{H_{F}^{f}(V, W)}{f}-\pi(V) \pi(W)+g\left(W,^{F} \nabla_{V} P\right)\right) X+\frac{(X h)}{h} \pi(W) V .
\end{aligned}
$$

From equation (2.5), we have

$$
\begin{aligned}
\tilde{R}(U, V) W= & R(U, V) W+g\left(W, \nabla_{U} P\right) V-g\left(W, \nabla_{V} P\right) U \\
& +g(U, W) \nabla_{V} P-g(V, W) \nabla_{U} P \\
& +\pi(P)(g(U, W) V-g(V, W) U) \\
& +(g(V, W) \pi(U)-g(U, W) \pi(U)) P \\
& +\pi(W)(\pi(V) U-\pi(U) V) .
\end{aligned}
$$

By use of Lemma 3.1 and Lemma 3.2 in above equation, we obtain

$$
\begin{aligned}
\tilde{R}(U, V) W= & { }^{F} \tilde{R}(U, V) W \\
& -\frac{\|\operatorname{grad} h\|^{2}}{h^{2}}(g(V, W) U-g(U, W) V) \\
& +(\pi(U) g(V, W)-g(U, W) \pi(V)) \frac{\operatorname{grad} h}{h} .
\end{aligned}
$$

Thus, we have completed the proof. 
As a consequence of Proposition 4.3 and Proposition 4.4, by a contraction of the curvature tensors, we obtain the Ricci tensors of the doubly warped product with respect to the semi-symmetric metric connection as follows:

Corollary 4.5. Let $M=B_{f} \times{ }_{h} F$ be a doubly warped product manifold and let $\tilde{S},{ }^{B} \tilde{S}$ and ${ }^{F} \tilde{S}$ be the Ricci tensors with respect to the semi-symmetric metric connections $\tilde{\nabla},{ }^{B} \tilde{\nabla}$ and ${ }^{F} \tilde{\nabla}$, respectively. If $X, Y \in \mathfrak{X}(B), V, W \in \mathfrak{X}(F)$ and $P \in \mathfrak{X}(B)$, then

$$
\begin{aligned}
\tilde{S}(X, Y)= & { }^{\tilde{S}}(X, Y)-\frac{n_{2}}{h} H_{B}^{h}(X, Y)+n_{2} \pi(X) \pi(Y)-n_{2} g\left(Y,{ }^{B} \nabla_{X} P\right) \\
& -\left(\left(n_{1}-1\right) \frac{\|\operatorname{grad} f\|^{2}}{f^{2}}+n_{2} \pi(P)+n_{2} \frac{P h}{h}+\frac{1}{f} \triangle f\right) g(X, Y), \\
\tilde{S}(X, V)= & \left(n_{1}-1\right) \frac{(V f)(X h)}{h f}+(n-2) \frac{(V f)}{f} \pi(X), \\
\tilde{S}(V, X)= & \left(n_{2}-1\right) \frac{(V f)(X h)}{h f}-(n-2) \frac{(V f)}{f} \pi(X), \\
\tilde{S}(V, W)= & { }^{F} S(V, W)-\frac{n_{1}}{f} H_{F}^{f}(V, W) \\
& -\left(\operatorname{div} P+\frac{1}{h} \triangle h+n_{1} \frac{P h}{h}+\left(n_{1}-1\right) \pi(P)\right) g(V, W) \\
& -\left(n_{2}-1\right)\left(\frac{\|\operatorname{grad} h\|^{2}}{h^{2}}+\frac{2 P h}{h}+\pi(P)\right) g(V, W) .
\end{aligned}
$$

Corollary 4.6. Let $M=B_{f} \times{ }_{h} F$ be a doubly warped product manifold and let $\tilde{S},{ }^{B} \tilde{S}$ and ${ }^{F} \tilde{S}$ be the Ricci tensors with respect to the semi-symmetric metric connections $\tilde{\nabla},{ }^{B} \tilde{\nabla}$ and ${ }^{F} \tilde{\nabla}$, respectively. If $X, Y \in \mathfrak{X}(B), V, W \in \mathfrak{X}(F)$ and $P \in \mathfrak{X}(F)$, then

$$
\begin{aligned}
\tilde{S}(X, Y)= & B S(X, Y)-n_{2} \frac{H_{B}^{h}(X, Y)}{h} \\
& -\left(n_{1}-1\right)\left(\frac{\|\operatorname{grad} f\|^{2}}{f^{2}}+\frac{2 P f}{f}\right) g(X, Y) \\
& -\left(\frac{1}{f} F \triangle f+(n-2) \pi(P)+n_{2} \frac{P f}{f}+\operatorname{div} P\right) g(X, Y), \\
\tilde{S}(X, V)= & \left(n_{1}-1\right) \frac{(V f)(X h)}{h f}-(n-2) \frac{X h}{h} \pi(V),
\end{aligned}
$$




$$
\begin{aligned}
\tilde{S}(V, W)= & { }^{F} \tilde{S}(V, W) \\
& -n_{1} g\left(W{ }^{F} \nabla_{V} P\right)-\frac{n_{1}}{f} H_{F}^{f}(V, W)+n_{1} \pi(V) \pi(W) \\
& -\left(\frac{1}{h}^{B} \triangle h+n_{1} \frac{P f}{f}+\left(n_{2}-1\right) \frac{\|\operatorname{grad} h\|^{2}}{h^{2}}+n_{1} \pi(P)\right) g(V, W) .
\end{aligned}
$$

As a consequence of Corollary 4.5 and Corollary 4.6, by a contraction of the Ricci tensors, we get scalar curvatures of the doubly warped product with respect to the semi-symmetric metric connection as follows:

Corollary 4.7. Let $M=B_{f} \times{ }_{h} F$ be a doubly warped product manifold and $P \in \mathfrak{X}(B)$. Let $\tilde{r},{ }^{B} \tilde{r}$ and ${ }^{F} \tilde{r}$ be the scalar curvatures with respect to the semi-symmetric metric connections $\tilde{\nabla},{ }^{B} \tilde{\nabla}$ and ${ }^{F} \tilde{\nabla}$, respectively. Then

$$
\begin{aligned}
\tilde{r}= & \frac{B \tilde{r}}{f^{2}}+\frac{F r}{h^{2}}-n_{1}\left(n_{1}-1\right) \frac{\|\operatorname{grad} f\|^{2}}{f^{2}}-n_{2}\left(n_{2}-1\right) \frac{\|\operatorname{grad} h\|^{2}}{h^{2}} \\
& -2 n_{2}(n-1) \frac{P h}{h}-2 n_{1} \frac{F \Delta f}{f}-2 n_{2} \frac{B \Delta h}{h}-2 n_{2} \operatorname{div} P \\
& -n_{2}\left(n+n_{1}-3\right) \pi(P) .
\end{aligned}
$$

Corollary 4.8. Let $M=B_{f} \times{ }_{h} F$ be a doubly warped product and $P \in$ $\mathfrak{X}(F)$. Let $\tilde{r},{ }^{B} \tilde{r}$ and ${ }^{F} \tilde{r}$ be the scalar curvatures with respect to the semisymmetric metric connections $\tilde{\nabla},{ }^{B} \tilde{\nabla}$ and ${ }^{F} \tilde{\nabla}$, respectively. Then

$$
\begin{aligned}
\tilde{r}= & \frac{{ }^{B} r}{f^{2}}+\frac{F \tilde{r}}{h^{2}}-n_{1}\left(n_{1}-1\right) \frac{\|\operatorname{grad} f\|^{2}}{f^{2}}-n_{2}\left(n_{2}-1\right) \frac{\|\operatorname{grad} h\|^{2}}{h^{2}} \\
& -2 n_{1}(n-1) \frac{P f}{f}-2 n_{1} \frac{F \Delta f}{f}-2 n_{2} \frac{{ }^{B} \Delta h}{h}-2 n_{1} \operatorname{div} P \\
& -n_{1}\left(n+n_{2}-3\right) \pi(P) .
\end{aligned}
$$

REMARK 4.9. Doubly warped product manifolds with a semi-symmetric metric connection has also been studied by Sular [20], where the author has taken the associated vector field $P \in \mathfrak{X}(M)$ as $P=P_{B}+P_{F}, P_{B}$ and $P_{F}$ are the components of $P$ on $B$ and $F$, respectively.

5. Einstein doubly warped product manifolds endowed with the semi-symmetric metric connection. In this section, we consider Einstein doubly warped products and Einstein-like doubly warped product of class $\mathcal{A}$ endowed with the semi-symmetric metric connection.

TheOrem 5.1. Let $M=B_{f} \times{ }_{h} F$ be a doubly warped product manifold $(n>2)$ and $P \in \mathfrak{X}(B)$. Then $(M, \tilde{\nabla})$ is an Einstein manifold with Einstein 
constant $\mu$ if and only if

$$
\begin{aligned}
{ }^{B} \tilde{S}(X, Y)= & \frac{n_{2}}{h} H_{B}^{h}(X, Y)-n_{2} \pi(X) \pi(Y)+n_{2} g\left(Y,{ }^{B} \nabla_{X} P\right) \\
& +\left(\left(n_{1}-1\right) \frac{\|\operatorname{grad} f\|^{2}}{f^{2}}+n_{2} \pi(P)+n_{2} \frac{P h}{h}+\frac{1}{f} \triangle f+\mu\right) g(X, Y),
\end{aligned}
$$

$$
\begin{aligned}
F S(V, W)= & \frac{n_{1}}{f} H_{F}^{f}(V, W) \\
& +\left(\operatorname{div} P+\frac{1}{h} \triangle h+n_{1} \frac{P h}{h}+\left(n_{1}-1\right) \pi(P)\right) g(V, W) \\
& +\left(n_{2}-1\right)\left(\frac{\|\operatorname{grad} h\|^{2}}{h^{2}}+\frac{2 P h}{h}+\pi(P)+\mu\right) g(V, W) .
\end{aligned}
$$

Proof. The proof follows from Corollary 4.5 and Corollary 4.6.

THEOREM 5.2. Let $(M, \tilde{\nabla})$ be an Einstein doubly warped product manifold with Einstein constant $\mu$ and $P \in \mathfrak{X}(B)$. Suppose that $B$ is a compact Riemannian manifold, $F$ is a complete Riemannian manifold, $n_{1}, n_{2} \geq 2$, and $H_{F}^{f}(V, W)$ is a constant multiple of $g_{F}$. If $\mu \leq 0, P h \geq 0, \pi(P) \leq 0$, div $P \leq 0$ then $M$ is a warped product manifold.

Proof. Since $H_{F}^{f}(V, W)$ is a constant multiple of $g_{F}$, then by using the result of Tashiro [23, Theorem 2], we can say that $F$ is a Euclidean space, then Ricci tensor of $F$ is zero. By (5.2), we get

$$
\begin{aligned}
0= & \left(\frac{c n_{1}}{f}+\left(n_{2}-1\right)\left(\|\operatorname{grad} h\|^{2}+2 h P h+h^{2} \pi(P)+\mu h^{2}\right)\right. \\
& \left.+h^{2} \operatorname{div} P+h \triangle h+n_{1} h P h+\left(n_{1}-1\right) h^{2} \pi(P)\right) g_{F}(V, W) .
\end{aligned}
$$

So

$$
\begin{aligned}
0= & \left(\frac{c n_{1}}{f}+\left(n_{2}-1\right)\left(\|\operatorname{grad} h\|^{2}+2 h P h+h^{2} \pi(P)+\mu h^{2}\right)\right. \\
& \left.+h^{2} \operatorname{div} P+h \triangle h+n_{1} h P h+\left(n_{1}-1\right) h^{2} \pi(P)\right),
\end{aligned}
$$

Let $x \in B$ such that $h(x)$ is the maximum of $h$ on $B$. Therefore $\operatorname{grad} h(x)=0$ and $\triangle h(x) \leq 0$. Then $P h(x)=g(\operatorname{grad} h(x), P)=0$. So the equation (5.3) at the point $x$ is

$$
\begin{aligned}
0= & \left(\left(n_{2}-1\right)\left(h^{2}(x) \pi(P)+\mu h^{2}(x)\right)\right. \\
& \left.+h^{2}(x) \operatorname{div} P+h(x) \triangle h(x)+\left(n_{1}-1\right) h^{2}(x) \pi(P)\right) .
\end{aligned}
$$


By eq(5.3) and eq(5.4), we have

$$
\begin{aligned}
& 0=\left(\frac{c n_{1}}{f}+\left(n_{2}-1\right)\left(\|\operatorname{grad} h\|^{2}+2 h P h+\left(h^{2}-h^{2}(x)\right) \pi(P)+\mu\left(h^{2}-h^{2}(x)\right)\right)\right. \\
& +\left(h^{2}-h^{2}(x)\right) \operatorname{div} P+h \triangle h-h(x) \triangle h(x)+n_{1} h P h \\
& \left.+\left(n_{1}-1\right)\left(h^{2}-h^{2}(x)\right) \pi(P)\right) .
\end{aligned}
$$

Since $\mu \leq 0, P h \geq 0, \pi(P) \leq 0$, $\operatorname{div} P \leq 0$, then equation (5.5) implies that $h \triangle h \leq 0$, which shows that the Laplacian has constant sign and hence $h$ is constant.

THEOREM 5.3. Let $(M, g)$ be an Einstein doubly warped product manifold $M=I_{f} \times{ }_{h} F$ with respect to the semi-symmetric metric connection, where $I$ is an open interval, $\operatorname{dim} I=1$ and $\operatorname{dim} F=n-1(n \geq 3)$. If $P \in \mathfrak{X}(I)$, then $f$ is constant on $F$ or $P h=h \pi(P)$.

ProOF. Let $(M, g)$ be a doubly warped product $M=I_{f} \times{ }_{h} F$, where $I$ is an open interval, $\operatorname{dim} I=1$ and $\operatorname{dim} F=n-1(n \geq 3)$. By Corollary 4.5, we have

$$
\begin{gathered}
\tilde{S}(X, V)=(n-2) \frac{V f}{f} \pi(X), \\
\tilde{S}(V, X)=(n-2) \frac{(V f)}{f}\left(\frac{X h}{h}-\pi(X)\right) .
\end{gathered}
$$

Since $M$ is an Einstein manifold with respect to the semi-symmetric metric connection, we can write

$$
\tilde{S}(P, V)=\alpha g(P, V)
$$

where $\alpha$ is constant. But $g(P, V)=0$, therefore $\tilde{S}(P, V)=0$. By (5.6), we have either $V f=0$ or $\frac{P h}{h}=\pi(P)$. Therefore, either $f$ is constant or $P h=h \pi(P)$.

Definition 5.4. [22] A Riemannian manifold $(M, g)$ is said to admit a cyclic-Ricci parallel tensor or is Einstein-like of class $\mathcal{A}$ if

$$
\left(\nabla_{X} S\right)(Y, Z)+\left(\nabla_{Y} S\right)(Z, X)+\left(\nabla_{Z} S\right)(X, Y)=0
$$

for any vector fields $X, Y, Z \in \mathfrak{X}(M)$ or equivalently $\left(\nabla_{X} S\right)(X, X)=0$.

Proposition 5.5. Let $M=B_{f} \times{ }_{h} F$ be an Einstein-like doubly warped product manifold of class $\mathcal{A}$ with respect to the semi-symmetric metric connection $\tilde{\nabla}$ and $P \in \mathfrak{X}(B)$. The Riemannian manifold $B$ is an Einstein-like 
manifold of class $\mathcal{A}$ with respect to the semi-symmetric metric connection $\tilde{\nabla}$ if and only if ${ }^{B} \nabla_{X} Y=\frac{X h}{2 h} Y,{ }^{B} \nabla_{X} P=0$ and

$$
\begin{aligned}
0= & n_{2} \pi(X)\left(\pi(X) \frac{X h}{h}-\frac{\|\operatorname{grad} h\|^{2}}{2 h^{2}} g(X, X)\right) \\
& +\frac{X h}{h} g(X, X)\left(\left(n_{2}-1\right) \frac{\|\operatorname{grad} f\|^{2}}{f^{2}}-n_{2} \pi(P)-n_{2} \frac{1}{f} \triangle f\right) .
\end{aligned}
$$

Proof. By using (4.1), (4.2) and Corollary 4.5, we have

$$
\begin{aligned}
\left(\tilde{\nabla}_{X} \tilde{S}\right)(X, X) & =X \tilde{S}(X, X)-\tilde{S}\left(\tilde{\nabla}_{X} X, X\right)-\tilde{S}\left(X, \tilde{\nabla}_{X} X\right) \\
= & X\left({ }^{B} \tilde{S}(X, X)-\frac{n_{2}}{h} H_{B}^{h}(X, X)+n_{2} \pi(X) \pi(X)-n_{2} g\left(X,{ }^{B} \nabla_{X} P\right)\right) \\
& -X\left(\left(\left(n_{1}-1\right) \frac{\|\operatorname{grad} f\|^{2}}{f^{2}}+n_{2} \pi(P)+n_{2} \frac{P h}{h}+\frac{1}{f} \triangle f\right) g(X, X)\right) \\
& -\tilde{S}\left(\tilde{\nabla}_{X}^{B} X-\frac{1}{f} g(X, X) \operatorname{grad} f, X\right)-\tilde{S}\left(X, \tilde{\nabla}_{X}^{B} X-\frac{1}{f} g(X, X) \operatorname{grad} f\right),
\end{aligned}
$$$$
\left(\tilde{\nabla}_{X} \tilde{S}\right)(X, X)=\left(\tilde{\nabla}_{X}^{B} \tilde{S}\right)(X, X)+\frac{n_{2}}{h^{2}} X h H_{B}^{h}(X, X)-\frac{n_{2}}{h} X\left(H_{B}^{h}(X, X)\right)
$$$$
+n_{2} X(\pi(X))^{2}-n_{2} X g\left(X,{ }^{B} \nabla_{X} P\right)
$$$$
-\left(\left(n_{1}-1\right) \frac{\|\operatorname{grad} f\|^{2}}{f^{2}}+n_{2} \pi(P)+n_{2} \frac{P h}{h}+\frac{1}{f} \triangle f\right) X g(X, X)
$$$$
-\left(\left(n_{1}-1\right) X \frac{\|\operatorname{grad} f\|^{2}}{f^{2}}+n_{2} X \pi(P)+n_{2} X \frac{P h}{h}+X \frac{1}{f} \triangle f\right) g(X, X)
$$$$
+\frac{1}{f} g(X, X) \tilde{S}(\operatorname{grad} f, X)+\frac{1}{f} g(X, X) \tilde{S}(X, \operatorname{grad} f) .
$$

Since ${ }^{B} \nabla_{X} Y=\frac{X h}{2 h} Y$ and ${ }^{B} \nabla_{X} P=0$, we have

$$
\begin{aligned}
\left(\tilde{\nabla}_{X} \tilde{S}\right)(X, X)= & \left(\tilde{\nabla}_{X}^{B} \tilde{S}\right)(X, X)+n_{2} \pi(X)\left(\pi(X) \frac{X h}{h}-\frac{\|\operatorname{grad} h\|^{2}}{2 h^{2}} g(X, X)\right) \\
& +\frac{X h}{h} g(X, X)\left(\left(n_{2}-1\right) \frac{\|\operatorname{grad} f\|^{2}}{f^{2}}-n_{2} \pi(P)-n_{2} \frac{1}{f} \triangle f\right) .
\end{aligned}
$$

The proof is completed.

REMARK 5.6. Einstein-like manifolds are natural extension of Einstein manifolds. Einstein-like manifolds admitting different curvature conditions were considered by Calvaruso [6]. Einstein-like manifolds of dimensions 3 and 
4 are studied in $[\mathbf{3}, \mathbf{5}]$. Projective spaces and spheres furnished with class $\mathcal{A}$ or class $\mathcal{B}$ Einstein-like metrics were classified in [17]. An interesting study in $[\mathbf{1 5}]$ shows that Einstein-like Generalized Robertson-Walker spacetimes are perfect fluid space-times except one class of Gray's decomposition.

Acknowledgements. We thank the reviewer for valuable suggestions and corrections.

\section{References}

1. Agaoka Y., Kim I.-B., Kim B.H., Yeom D.J., On doubly warped product manifolds, Mem. Fac. Integrated Arts \& Sci., Hiroshima Univ. Ser. IV, 24 (1998), 1-10.

2. Allison D.E., Pseudocovexity in Lorentzian doubly warped products, Geom. Dedicata, 39 (1991), 223-227.

3. Berndt J., Three-dimensional Einstein-like manifolds, Differential Geom. Appl., 2 (1992), 385-397.

4. Bishop R.L., O'Neill B., Manifolds of negative curvature, Trans. Amer. Math. Soc., 145 (1969), 1-49.

5. Bueken P., Vanhecke L., Three and four dimensional Einstein-like manifolds and homogeneity, Geometriae Dedicata, 75 (1999), no. 2, 123-136.

6. Calvaruso G., Einstein-like metrics on three-dimensional homogeneous Lorentzian manifolds, Geom. Dedicata, 127 (2007), 99-119.

7. Friedmann A., Schouten J.A., Über die Geometrie der halbsymmetrischen Übertragungen, Math. Z., 21 (1924), no. 1, 211-223.

8. Gebarowski A., Doubly warped products with harmonic Weyl conformal curvature tensor, Colloq. Math., 67 (1994), 73-89.

9. Gebarowski A., On conformally recurrent doubly warped products, Tensor (N.S.), 57 (1996), 192-196.

10. Gupta P., On compact Einstein doubly warped product manifolds, Tamkang Jour. Math., 49 (2018), no. 1, 267-275.

11. Hatzinikitas A.N., A note on doubly warped product spaces, arXiv:1403.0204v1.

12. Hayden H.A., Subspace of a space with torsion, Proc. London Math. Soc., II Series, 34 (1932), 27-50.

13. Imai T., Hypersurfaces of a Riemannian manifold with semi-symmetric metric connection, Tensor (N.S.), 23 (1972), 300-306.

14. Imai T., Notes on semi-symmetric metric connections, Commemoration volumes for Prof. Dr. Akitsugu Kawaguchi's seventieth birthday, Vol. I. Tensor (N.S.), 24 (1972), 293-296.

15. Mantica C.A., Molinari L.G., Young J.S., Shenawy S., Perfect-Fluid, Generalized Robertson-Walker Space-times, and Gray's Decomposition, J. Math. Phys., 60 (2019), no. 5, 052506, 9 pp.

16. O'Neill B., Semi-Riemannian geometry with applications to relativity, Academic Press, New York, London, 1983.

17. Peng C., Chao Q., Homogeneous Einstein-like metrics on spheres and projective spaces, Differential Geometry and its Applications, 44 (2016), 63-76.

18. Ponge R., Reckziegel H., Twisted products in pseudo-Riemann geometry, Geometriae Dedicata, 49 (1993), 15-25.

19. Ramos M.P.M., Vaz E.G.L.R., Carot J., Double warped space time, Jour. Math. Phys., 44 (2003), no. 10, 4835-4869. 
20. Sular S., Semi-symmetric metric connection on doubly warped product manifolds, Int. Jour. Pure Math., 4 (2017), 52-58.

21. Sular S., Ozgur C., Warped product with semi-symmetric metric connection, Taiwanese Journal of Mathematics, 15 (2011), no. 4, 1701-1719.

22. Sumitomo T., On a certain class of Riemannian homogeneous spaces, Coll. Math., 26 (1972), 129-133.

23. Tashiro Y., Complete Riemannian manifolds and some vector fields, Transaction of the American Mathematical Society, 117(1965), 251-275.

24. Tripathi M.M., A new connection in a Riemannian manifold, Int. Electron. J. Geom., 1 (2008), no. 1, 15-24.

25. Ünal B., Doubly warped products, Diff. Geom. Appl., 15 (2001), no. 3, 253-263.

26. Yano K., On semi-symmetric metric connections, Rev. Roumaine Math. Pures Appl., 15 (1970), 1579-1586.

Received September 27, 2019

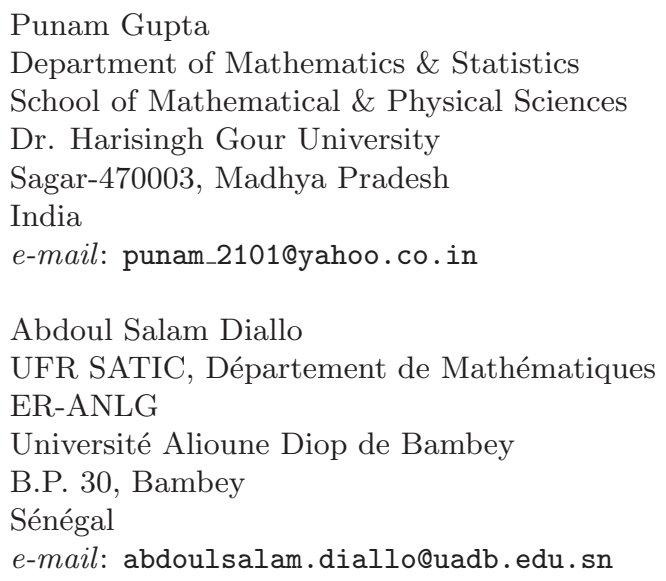

